\title{
The Contents of Some Mathematical Courses need to be Improved: a Viewpoint for Education Reformation
}

\author{
Wei Wang \\ Dept. of Mathematics, School of Information \\ Renmin University of China \\ Beijing, China \\ wwei@ruc.edu.cn
}

\begin{abstract}
Mathematics is the language for natural sciences, engineering technology, or social sciences. In this paper, after analyzing the problems of recent mathematical education, and based on the conclusion that mathematics is not only just an abstract subject, but also an economic and effective methodology, we propose a viewpoint on the reformation on the contents of some basic courses for mathematical education, especially on the improvement to the contents of some main mathematical courses. We propose the possible extension for mathematical analysis, advanced algebra, and mathematical statistics from the viewpoint of education reformation, and emphasize the necessary of employing the information technology in mathematical education.
\end{abstract}

Keywords-mathematical education; education reformation; basic course; content improvement

\section{INTRODUCTION}

When I was an undergraduate student of mathematics, I was asked what did you learn about? I told them they are about the methods and theory considered at 17 th century. More than 30 years past, when I face the undergraduate students today, they still learn almost the same things as I did. Does it mean that there is anything more needs to be studied. The answer is not. The problem is that the textbooks haven't been improved. In fact, those textbooks cannot meet the requirements of the modern education and social development. That is why some people complain about the uselessness of math. From the education reformation of math, what should be done? This paper will provide certain kinds of consideration on the improvement to the contents of some mathematical courses.

It is known that, math is the study of topics such as quantity (numbers), and structure, space (geometry). There is a range of views among mathematicians and philosophers as to the exact scope and definition of math. Through the use of abstraction and logic, mathematics developed from counting, calculation, measurement to the systematic study of the shapes and motions of physical objects. When mathematical structures are good models of real phenomena, then mathematical reasoning can provide insight or predictions about nature ([1], [2]).

Meanwhile, the problems of mathematics arise from many different kinds of practical requirements. At first these were found in commerce, land measurement, architecture and later astronomy. Today, all sciences and technology suggest problems studied by mathematicians, and many problems arise within mathematics itself. For example, the physicist Richard Feynman invented the path integral formulation of quantum mechanics using a combination of mathematical reasoning and physical insight, and today's string theory, a still-developing scientific theory which attempts to unify the four fundamental forces of nature, continues to inspire new math ([2], [3]).

Unfortunately, the textbooks appeared to students are that math concerns about classic problems, and it is the subject with the features of beauty, precision, abstract, and unassailable. They are full of algorithms and skills. And in the textbooks, little is related with today's development and the requirements.

In this paper, we consider the problem on the reformation for the contents of main mathematical courses for math education, and the improvement of some mathematical textbooks or courses, such as mathematical analysis, advanced algebra and mathematical statistics, and so on. Firstly, after analyzing the characteristics on the status of recent mathematical textbooks and the relevant courses, we provide some solutions on the modification of main mathematical courses. Secondly, in order to solve the problem encounter for the development of sciences, we will provide some consideration for the improvement of the contents for the courses mention above, we extremely support to introduce the information method for education reformation, we propose the possible extension for those courses.

\section{MATHEMATICAL ANALYSIS SHOULD BE EXTENDED}

There are several branches in math. Mathematical analysis is a fundamental branch of math that studies continuous change and includes the theories of limits, continuity, differentiation, integration, measure, infinite series, and analytic functions.

\section{A. The background and history of mathematical analysis}

Although mathematical analysis developed in the 17th century, many of its ideas can be traced back to earlier mathematicians. Early results in analysis were implicitly present in the early days of ancient Greek math. Later, Greek mathematicians made more explicit use of the concepts of limits and convergence when they used the method of exhaustion to compute the area and volume of regions and 
solids. The explicit use of infinitesimals appears in Archimedes' The Method of Mechanical Theorems. In Asia, the Chinese mathematician Hui Liu, used the method of exhaustion in the 3 rd century $\mathrm{AD}$, to find the area of a circle. Chongzhi $\mathrm{Zu}$ established a method, which is called Cavalieri's principle, to find the volume of a sphere in the 5th century ([4]).

The modern foundations of mathematical analysis were established in Europe during the 17th century. Descartes and Fermat independently developed analytic geometry, and a few decades later, Newton and Leibniz independently developed infinitesimal calculus, which grew into analysis topics such as the calculus of variations, ordinary and partial differential equations, Fourier analysis, and generating functions. During this period, calculus techniques were applied to approximate discrete problems by continuous ones ([4] and references there)

In the 18th century, Euler introduced the notion of mathematical function. When Bernard Bolzano introduced the modern definition of continuity in 1816 , real analysis began to emerge as an independent subject. In 1821, Cauchy began to put calculus on a firm logical foundation by rejecting the principle of the generality of algebra, which was widely used in earlier work, particularly by Euler. Instead, Cauchy formulated calculus in terms of geometric ideas and infinitesimals. He also introduced the concept of the Cauchy sequence, and started the formal theory of complex analysis. Meanwhile, Poisson, Liouville, Fourier and others studied harmonic analysis ([4]).

In the middle of the 19th century Riemann introduced his theory of integration. The last third of the century saw the arithmetization of analysis by Weierstrass, who thought that geometric reasoning was inherently misleading, and introduced the "epsilon-delta" definition of limits, thus founding the modern field of mathematical analysis. Then, mathematicians started worrying that they were assuming the existence of a continuum of real numbers without proof. Dedekind then constructed the real numbers, which serve to fill the "gaps" between rational numbers, thereby creating a complete set: the continuum of real numbers. Around that time, the attempts to refine the theorems of Riemann integration led to the study of the 'size' of the set of discontinuities of real functions.

Rigorous arguments first appeared in Greek math, most notably in Euclid's Elements. Since the pioneering work of Giuseppe Peano, David Hilbert, and others on axiomatic systems in the late 19th century, it has become customary to view mathematical research as establishing truth by rigorous deduction from appropriately choosing axioms and definitions. Math developed at a relatively slow pace until the Renaissance, when mathematical innovations interacting with new scientific discoveries, which led to a rapid increase in the rate of mathematical discovery that has continued to the present day.

These theories are usually studied in the context of real and complex numbers and functions. Analysis evolved from calculus, which involves the elementary concepts and techniques of analysis. With the efforts of mathematicians, analysis may be distinguished from geometry. However, it can be applied to any space of mathematical objects that has a definition of nearness (a topological space) or specific distances between objects (a metric space).
In the branch of mathematical analysis, also known as advanced calculus, it mainly concerns the methods to describe the most basic characteristics related with changes or evolutions, such as the changing speed and cumulative effect, which are the most fundamental problems in philosophy. It is the introduction on the concept of limits makes calculus a significant discovery of human beings. That makes the computation of many complex problems effectively and easily, i.e., Calculus is not just a subject of math, it also provides an effective methodology for complex computing, such as the computing of area or volume with irregular shapes, etc ([5]). At the same time, it provides useful results related with other problems, for example, Talyor's formula is a useful result for the approximation of functions, and it is a tool for prediction.

\section{B. The drawback and imporovement of the relevent textbooks}

From the textbooks used for mathematical analysis we know that many of the contents concern about calculus. And the calculus is about the problem of derivative or differential and integration or integral. These are all come from the classical geometrical or physical problem, such as, the computation of displacement, velocity, force, and energy, etc. The reason is that the mathematician approaches a problem set by the needs of physics, as when the physicist or the engineer asks of him the calculation of some number in view of an application.

At the same time, in the end of the 20th century, with the need for random phenomena, the analytic method on stochastic processes was proposed. The main result is the Ito integration. It plays an important role for the analysis of financial math. Such kind of knowledge is also the important development of analysis, but it cannot be seen in the usual analytic textbooks.

Recently, the requirements of information processing, and analyzing of complex problems related with society and economics are also the ones that mathematics will encountered. There are some others requirements for the mathematical analysis, such as, financial analysis, information fusion, and social analysis, network computing, and so on.

Unfortunately, many of the textbooks on mathematical analysis concern little about modern requirements. From the viewpoint of education reformation, we have the suggestions as follows.

Firstly, we should try our best to let students to know the background and the history of mathematical analysis, and to understand the essence and usefulness of mathematical analysis for all the other subjects. Let the students to understand the new requirements of analysis, and try to tell them how to solve the problem based on the ideology of mathematical analysis.

Secondly, we should also let students to know that the concepts and the examples to indicate the effective and efficiency of analysis are still many. For example, with the introduction of Brownian motion, even the introduction of diffusion process, the evolutional processes of complex phenomena can be modeled properly. In recent decades, the research of financial problems, the prices of financial markets can be described by Brownian motion. Meanwhile, based on conclusions about the evolution of polymers, the model on the growth of individuals or even the evolution of social groups 
can be built ([6]). They belong to the field of analysis. They should be included in the textbooks.

Thirdly, because some of contexts in the textbooks can be solved with the use of information technology, for example, the plotting of a function, the proof of existence of differential equations, etc. so many of the contents in the conventional textbooks can be replaced by the improved ones. At the same time, certain kinds of differential equations can also be solved by programming, i.e. the solution of differential equations can be obtained by programming. As a matter of fact, many mathematical problems can be solved by using software, and there is some effective software, for instance, Matlab or Mathematica are tools for solving mathematical problems.

\section{Algebra Also NeEDS to Be IMPROVED}

Another important branch of mathematics is algebra. Algebra is really an abstract subject of math. Linear algebra is the sub-branch of algebra concerning vector spaces and linear mappings between linear spaces. It includes the study of lines, planes, and subspaces, it also concerns with properties common to all vector spaces (see [7] and reference therein).

The set of points with coordinates that satisfy a linear equation form a hyperplane in an $n$-dimensional space. The conditions under which a set of $n$ hyperplanes intersect in a single point is an important focus of study in linear algebra. Linear algebra is central to both pure and applied mathematics. For instance, abstract algebra arises by relaxing the axioms of a vector space, leading to a number of generalizations.

Such an investigation is initially motivated by a system of linear equations containing several unknowns. Such equations are naturally represented using the formalism of matrices and vectors. Combined with calculus, linear algebra facilitates the solution of linear systems of differential equations, which is a conventional method in the linear system theory ([7]).

Techniques from linear algebra are also used in analytic geometry, engineering, physics, natural sciences, computer science, computer animation, and the social sciences (particularly in economics). Because linear algebra is such a well developed theory, nonlinear mathematical models are sometimes approximated by linear models. And the method of differential geometry is an extension of the method above.

The essence of linear algebra can be summarized as follows. It is the introduction of signs and the operations on the signs that the expression of certain results will be simple. Therefore, algebra is also an effective tool of complex computing for structure, such as vector, matrix, or even grid, etc. With the introduction of signs, and the introduction of computing rules for those signs, we can improve our computing effectively. The efficiency can be demonstrated on the solution of linear algebra equations, especially with the operation of inverse metrics, the solution can be expressed in a very simple form.

The use of transformation is another feature of math. It can make a circle into a square, a ball into a cube, and even some irregular shapes into regular ones. The important of all is that it makes calculation or processing easier.
From the viewpoint of education reformation on algebra, we have the suggestion as follows.

Firstly, we should try to let students to know that the essence of algebra. It is an abstract of the operations on numbers. It can be regarded as the operation on certain signs. It is also an abstract operation of structure, such as matrix operation. With the development of algebra, the structure can be extended, such as $\sigma$-algebra, which is used for random events in probability theory. Nowadays, there are some other requirements for the computing of structure, such as for the computation of tables or even networks, information processing of big data, etc.

Secondly, we should let the students to understand that transformation can give the same name to things with different material but similar form. The concept of homogeneous or homeomorphism is the example. From the transformation we can also find same characteristics of things with different material or different shapes, i.e., the similar things can be with the same mode. They make us perceive the essence or gist of many mathematical demonstrations. It is the methodology of homogeneous that we can regard the structure of a man's head as that of the planet on which we live. We can also regard the molecular motion as the evolution of society, etc.

Thirdly, we would better try to introduce new structure or transformations ([8]). For example, quantum mechanics is highly inspired by notions in linear algebra. In quantum mechanics, the physical state of a particle is represented by a vector, and observables, such as momentum, energy, and angular momentum, are represented by linear operators on the underlying vector space ([3]). So introduction new structure and new operations for the structure will be necessary for improving the textbooks. All these aspects should be included in the textbooks.

\section{How ABOUt MAthematical Statistics?}

Mathematical statistics is the branch of mathematics devoted to the study of mathematical methods for the organization, processing and utilization of statistical data for the inference and prediction of scientific and practical conclusions.

\section{A. The functions and methods of mathematical statistics}

Mathematical statistics is the application of math to statistics, which was originally conceived as the science of the state, the collection and analysis of facts about a country: its population, military, economy, land, and so forth. Gradually, it has been applied in almost all areas relating to quantitative analysis ([9]).

Statistical science is concerned with the planning of studies, especially with the design of randomized experiments and with the planning of surveys using random sampling.

There are many aspects in the mathematical statistics. The basic branches are the descriptive statistics and inference statistics. Descriptive statistics concerns about the methods to describe a sample, and inferential statistics infers predictions about a population that the sample represents, i.e. it is the process of drawing conclusions from data that are subject to random variation. Initial requirements of such procedures for inference and induction are that the process should produce reasonable answers when applied to wel-defined situations and 
that it should be general enough to be applied across a range of situations. Inferential statistics is used to test hypotheses, make estimations and predict the future trends by using sample data.

The common features of the statistical method in various areas of knowledge come down to the calculation of the number of objects in some group or other, the discussion of the distribution of quantitative attributes, the application of the sampling method, where a detailed investigation of an extensive collection is difficult, and the use of probability theory to estimate the adequacy of a number of observations for this or that conclusion, etc.

With the development and elaboration of the general ideas of mathematical statistics, there have been evolved various specialized branches, such as, covariance analysis, dispersion analysis, multi-dimensional statistical analysis, the statistical analysis of stochastic processes, regressive analysis, and factor analysis, etc. New considerations in regression analysis have appeared see also stochastic approximation. A major part in problems of mathematical statistics is played by the Bayesian approach to statistical problems.

The scientific methods mentioned above can also be used for many other subjects, such as social and economical analysis, especially, based on using the method of probability theory and mathematical statistics for economics, we can obtain the important inference for decision, and something likes that.

Statistical methods are useful and effective. But the data processing methods are complex. That is why the popularity of some effective software, such as SAS, SPSS, or E-views, etc. Those softwares are also useful for education.

\section{B. The problems and suggestions in mathematical statistics}

The methods of parameter estimation and hypothesis testing are based on the assumption that the number of observations required to attain a given precision in the conclusions is determined in advance. However, frequently an a priori determination of the number of observations is inconvenient. Since by not fixing the number of trials in advance, but by determining it during the experiment, it is possible to decrease the expected number of trials. In problems on parameter estimation the final decisions are numbers, i.e. the values of the estimators. On the other hand, in problems on hypotheses testing they are the accepted hypothesis, and the aim of the theory is to give rules for the acceptance of decisions which minimize the mean loss or risk ([9]).

The development of the methods of sequential analysis led, on one hand, to the study of controlled stochastic processes, and, on the other hand, to the appearance of statistical decision theory. This theory arises because the results of sequentially carrying out observations serve as a basis for the adoption of certain decisions.

The shortcomings on conventional statistic methods are as follows: the inference results are based on the analysis of the observable factors and the sample there lacks of dynamic regulation to the model based on the sample. So there are many aspects need to be improved. And there are still a lot of new fields and the new algorithms need to be explored. From the viewpoint on the education reformation of mathematical statistics, we have the suggestions as follows.

Firstly, we should try to let students to understand the thinking of statistics, and to have the ability to translate the practical problems into the relevant statistical problems. And then we can solve the problems by using certain softwares.

Secondly, it is better to let students to know that time series analysis is the method to build a model and to make statistical inference based on historical records. Therefore the model will depend on the choice of sample data and the modeling method. Owing to the complexity and uncertainty of practical problems, the characteristics of time series will probably be varied in coming periods. To improve the adaptability of the model, a method is proposed based on introducing dynamic innovation mechanism to the model ([10]).

Thirdly, in recent years, a novel modelling method for time series is considered. Because the influences of the internal or external factors are often inevitable, which may result in the irregular change of the series, especially in many of the financial time series, the conventional modelling methods cannot meet the requirements, or have a passive response to the factors. To find an effective method which can reflect the intrinsic essence or external influences of the series, the internal model principle, which is used in the synthesis of control systems for revealing the fundamental features of the factors, is introduced. It may open up broad prospects for improving the fitness or prediction ability of the models under the circumstances mentioned above ([11]). All these methods mentioned above should be included in the textbooks.

\section{CONCLUSIONS}

In this paper, we provide some solutions to the reformation on mathematical education, and we propose the possible improvements on the contents for some main courses, such as mathematical analysis, advanced algebra and mathematical statistics from the viewpoint of education reformation.

\section{REFERENCES}

[1] H. Poincare, The future of mathematics, at the general session of the Fourth International Congress of Mathematicians, Rome, April 6-11, 1908 .

[2] L.Mata, V. Monteiro, and F. Peixoto, "Attitudes towards Math: Effects of Individual, Motivational, and Social Support Factors," Hindawi Publishing Corporation Child Development Research, ”vol. 2012, 2012.

[3] G. Fraser, The New Physics for the Twenty-First Century, second edition, Cambridge University Press, 2006.

[4] Mathematical analysis, From Wikipedia, the free encyclopedia, https:// en.wikipedia.org/wiki/Mathematical_analysis

[5] W. Wang, "Math isn't just an abstract subject but an economic and efective methodology," in Engineering Technology, Engineering Education and Engineering Management, CRC Press, pp. 151-154, 2015

[6] W. Wang, "The conceptual models for the growth of individuals based on the viewpoint of philosophy," in Proc. the 2014 Asia-Pacific Humanities and Social Sciences Conference, pp. 808-814. Nov. 29-30, 2014 in Shanghai, China.

[7] Linear algebra, From Wikipedia, the free encyclopedia https: //en.wikipedia.org/wiki/Linear_algebra

[8] L L Cavalli Sforza, Genes, Peoples, and Languages, New York: North Point Press, 2000. 
[9] Encyclopedia of Mathematics, https://www.encyclopediaofmath.org /index.php/Main_Page

[10] W. Wang, "The method on improving the adaptability of time series models based on dynamical innovation," Communications in Computer and Information Sciences, vol. 288, pp. 210-217, 2012.
[11] W. Wang, "The novel modelling method of time series based on the internal model principle," Advances in Intelligent Systems Research, vol. 123, pp. 573-576, 2015. 